EPJ Web of Conferences 28, 12059 (2012)

DOI: $10.1051 /$ epjconf/20122812059

(C) Owned by the authors, published by EDP Sciences, 2012

\title{
Photon energy scale determination and commissioning with radiative $\mathbf{Z}$ decays
}

Olivier Bondu ${ }^{\mathrm{a}}$

Université de Lyon, Université C. Bernard Lyon 1, CNRS/IN2P3, Institut de Physique Nucléaire de Lyon, Villeurbanne, France

\begin{abstract}
The CMS electromagnetic calorimeter (ECAL) is composed of 75848 lead-tungstate scintillating crystals. It has been designed to be fast, compact, and radiation hard, with fine granularity and excellent energy resolution. Obtaining the design resolution is a crucial challenge for the SM Higgs search in the two photon channel at the LHC, and more generally good photon calibration and knowledge of the photon energy scale is required for analyses with photons in the final state. The behavior of photons and electrons in the calorimeter is not identical, making the use of a dedicated standard candle for photons, complementary to the canonical highyield $\mathrm{Z}$ decay to electrons, highly desirable.

The use of $Z$ decays to a pair of muons, where one of the muons emits a Bremsstrahlung photon, can be such a standard candle. These events, which can be cleanly selected, are a source of high-purity, relatively high-pt photons. Their kinematics are well-constrained by the $\mathrm{Z}$ boson mass and the precision on the muon momenta, and can be used for numerous calibration and measurement purposes. This proceeding presents the event selection method and the results of the photon energy scale measurement via $Z^{0} \rightarrow \mu \mu \gamma$ events as well as their use in evaluating the efficiency of photon identification requirements, based on data recorded by the CMS experiment in 2010 .
\end{abstract}

\section{Introduction}

\subsection{The Compact Muon Solenoid (CMS)}

The CMS experiment is one of the two multi-purpose experiments recording the collisions produced by the Large Hadron Collider (LHC), located at CERN, Geneva. Its main features are:

- its superconducting solenoid, generating a $3.8 \mathrm{~T}$ magnetic field,

- its hermicity and compactedness: 14000 tons, $15 \mathrm{~m} \mathrm{di-}$ ameter and $28.7 \mathrm{~m}$ long,

- its muon chambers.

A more detailed description can be found in [1]

\subsection{The electromagnetic calorimeter (ECAL)}

The ECAL of CMS has been designed to be fast, compact, radiation hard, with fine granularity and excellent energy resolution.

\subsubsection{Choice of $\mathrm{PbWO}_{4}$}

Several considerations guided the choice of $\mathrm{PbWO}_{4}$ as a material for the crystals:

- the Lead Tungstate has high density $\left(8.28 \mathrm{~g} / \mathrm{cm}^{3}\right)$, short radiation length $\left(X_{0}=0.89 \mathrm{~cm}\right)$ and small Molière radius $(2.2 \mathrm{~cm})$

a e-mail: olivier.bondu@cern.ch
- good energy resolution (longitudinal homogeneity),

- fast: $80 \%$ of the scintillation light is emitted in $25 \mathrm{~ns}$,

- radiation hard: changes in crystal opacity are measured by a dedicated laser monitoring system

\subsubsection{Layout}

The calorimeter is composed of 75848 scintillating crystals of $\mathrm{PbWO}_{4}$ :

- the barrel is composed of 36 supermodules of 1700 crystals each, with the scintillating light collected and converted by avalanche photo-diodes,

- the endcaps are composed of 4 Dees of 3662 crystals each, with the scintillating light collected and converted by vacuum photo-triodes.

In front of the ECAL endcaps, two layers of silicon detectors have been placed, in order to help discriminating between photons and neutral hadrons: the preshower.

The ECAL layout is illustrated in figure 1.

\section{Physics performance: motivation for a dedicated photon standard candle}

\subsection{ECAL calibration scheme}

The ECAL resolution has been measured in electron testbeams. It has been parametrized as follows:

$$
\frac{\sigma(E)}{E}=\frac{2.8 \%}{\sqrt{E(G e V)}} \oplus \frac{12 \%}{E(G e V)} \oplus 0.3 \%
$$




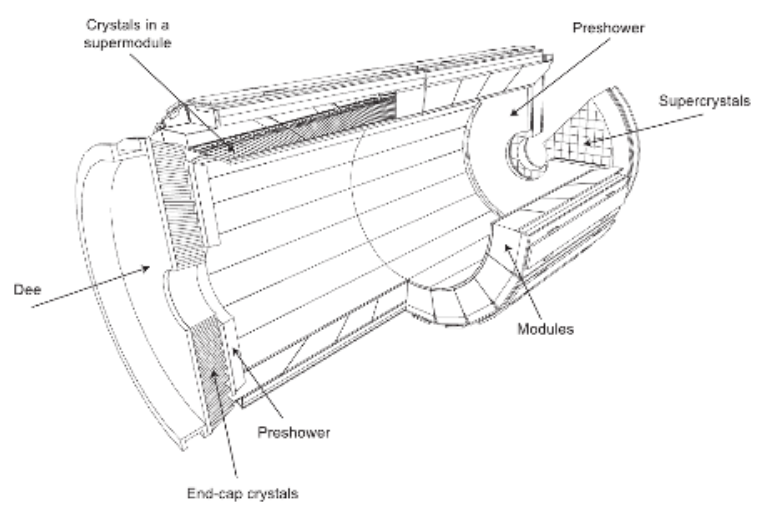

Fig. 1: The electromagnetic calorimeter (ECAL) of the CMS experiment.

The three contributions correspond respectively to the stochastic component (S), the electronic and experimental noise $(\mathrm{N})$, and a constant term $(\mathrm{C})$.

For photons of energy $100 \mathrm{GeV}(H \rightarrow \gamma \gamma$ range), the energy resolution is dominated by the constant term: the contribution of calibration to the energy resolution is significant. [2]:

The reconstructed energy of a particle in the ECAL is

$$
E_{e, \gamma}=F_{e, \gamma}(\eta) \cdot \sum_{\text {clustercrystals }} G(G e V / A D C) \cdot S_{i}(T, t) \cdot c_{i} \cdot A_{i}
$$

Where:

- $A_{i}$ represents a reconstructed amplitude in ADC counts,

- $c_{i}$ is an intercalibration constant,

- $G$ is the ECAL energy scale,

- $S_{i}$ is the correction for crystal transparency loss $T$ as a function of time $t$

- $F$ represents the object dependent energy correction. It depends on the particle type, energy, and pseudorapidity $\eta$, and also contains the shower containment corrections, geometry and material effects.

Different physics channels are available to evaluate the different calibration terms: $\pi^{0} \rightarrow \gamma \gamma, \eta \rightarrow \gamma \gamma, J / \psi \rightarrow$ $e^{+} e^{-}, W^{ \pm} \rightarrow e^{ \pm} v, Z^{0} \rightarrow e^{+} e^{-}$and symmetry around the $\phi$ axis of minimum bias events.

Good knowledge and control of intercalibration of the crystal response, photon energy scale and resolution are desirable for analyses with photons in the final state. This is especially crucial for $H \rightarrow \gamma \gamma$ searches. Since photons and electrons behave differently in the ECAL, there is a need for a dedicated standard candle for photons, in addition to $Z^{0} \rightarrow e^{+} e^{-}$.

\subsection{Radiative $Z^{0}$ decays, process selection strategy}

The $\mathrm{Z}$ decay to muons with final state radiation (FSR), where one of the muons emits a Bremsstrahlung photon (figure 2a), is interesting for several reasons:

- as the $\mathrm{Z}$ decays to muons, there are no other particles from the hard event in the ECAL apart from the photon, which allows a clean observation of photons,

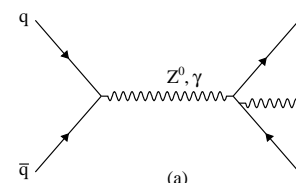

(a) FSR process

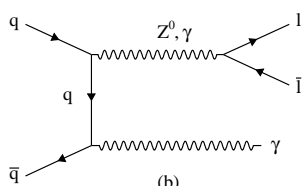

(b) ISR process
Fig. 2: Feynman diagrams of FSR and ISR processes.

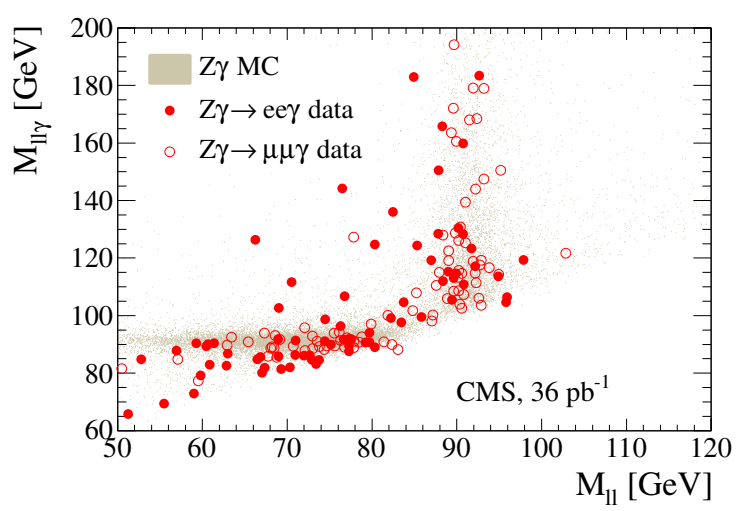

Fig. 3: Events featuring one photon and two leptons in the final state in the $M_{l l y}$ vs. $M_{l l}$ plane, from [3]. One can see two main contributions: ISR events where the dilepton invariant mass is around the $Z^{0}$ mass, and FSR events where the three-body invariant mass is around the $Z^{0}$ mass

- CMS delivers high precision measurement of muon momentum, and the $\mathrm{Z}$ boson properties are known from LEP measurements with high accuracy: these constrain the photon kinematics,

- this process is purely an electroweak decay, it then provides a clear and neat signal in hadronic collisions.

The main background process faking FSR decays are initial state radiations (ISR), as represented on figure $2 b$.

Several criteria are applied to select FSR decays:

- the standard CMS tight muon identification, without calorimetric isolation, is applied,

- the dimuon invariant mass is required to reject nonradiative $Z$ decays (see figure 3 ),

- the photon object selection remains as loose as possible: only fiducial cuts are required,

- some maximum angular separation between the photon and the closest muon is required to reject initial state radiation events,

- the three-body invariant mass $m_{\mu \mu \gamma}$ is required to be around the $Z^{0}$ mass.

Such selected events are a source of high-purity photons, with a steeply falling photon energy spectrum. As their kinematics are well-constrained by the $Z^{0}$ boson mass and the precision on the muon momenta, they can be used for numerous calibration and measurement purposes [4]. 


\section{Results}

\subsection{Photon Commissioning: $R_{9}$}

The variable $R_{9}$ is defined as [5]:

$$
R_{9}=\frac{E^{3 \times 3}}{E^{\text {SuperCluster }}}
$$

It quantifies the lateral width of an electromagnetic shower and is widely used within the CMS collaboration to distinguish converted and unconverted photons.

The tracker material in front of the calorimeter can cause the photons to convert, and in the strong magnetic field of the solenoid, the energy of the converted photons can spread in $\phi$. Clustering algorithms have been designed [4] to collect the energy of the photons, and have been optimized to give the best photon resolution. Thus these algorithms behave differently if a deposit is thought to belong to a converted photon, by means of a threshhold on the $R_{9}$ variable.

The limit setting process in the $H \rightarrow \gamma \gamma$ search [6, 7] is performed in resolution classes. The uncertainty in class assignment / migration between classes is a source of systematic error. As the $R_{9}$ variable exhibits a different behaviour for photons and for electrons, it is quantified with $Z^{0} \rightarrow \mu \mu \gamma$ events, as presented in Table 1 .

Table 1: Summary of systematic uncertainties on the $H \rightarrow$ $\gamma \gamma$ signal applicable to individual photons [7].

\begin{tabular}{|c|c|c|}
\hline Source & \multicolumn{2}{|c|}{ Uncertainty } \\
\hline $\begin{array}{r}\text { Photon identification efficiency } \\
\text { barrel } \\
\text { endcap }\end{array}$ & \multicolumn{2}{|c|}{$\begin{array}{l}1.0 \% \\
2.5 \%\end{array}$} \\
\hline $\begin{array}{lr}R_{9}>0.94 \text { efficiency } & \\
\text { (results in class migration) } & \text { barrel } \\
\text { endcap }\end{array}$ & \multicolumn{2}{|c|}{$\begin{array}{c}4 \% \\
6.5 \%\end{array}$} \\
\hline \multirow{2}{*}{ Energy resolution $\left(\Delta \sigma / E_{M C}\right)$} & $R_{9}>0.94$ & $R_{9}<0.94$ \\
\hline & $\begin{array}{l}0.2 \% \\
0.5 \%\end{array}$ & $\begin{array}{l}0.4 \% \\
0.4 \%\end{array}$ \\
\hline $\begin{array}{r}\text { Energy scale }\left(\left(E_{\text {data }}-E_{M C}\right) / E_{M C}\right) \\
\text { barrel } \\
\text { endcap }\end{array}$ & $\begin{array}{l}0.1 \% \\
0.3 \%\end{array}$ & $\begin{array}{l}0.4 \% \\
0.4 \%\end{array}$ \\
\hline
\end{tabular}

\subsection{Photon identification: lepton veto}

For the $H \rightarrow \gamma \gamma$ analyses [6,7], the efficiency of photon identification is measured in data using tag-and-probe techniques. $Z^{0} \rightarrow e^{+} e^{-}$events are used to determine the efficiency of the complete selection with the exception of the electron veto cut.

$Z^{0} \rightarrow \mu \mu \gamma$ events have been used to measure the efficiency for photons to pass the electron veto, with tag-andprobe techniques with the dimuon system as the tag and the photon candidate as the probe. The results are presented in Table 2.
Table 2: Photon identification efficiencies measured in the 4 photon categories (1, 3 - for $R_{9}>0.94$ barrel, endcap; 2 , 4 - for $R_{9}<0.94$ barrel, endcap) using tag and probe with $Z \rightarrow e e$ events (for all cuts except electron rejection) and with $Z \rightarrow \mu \mu \gamma$ (for the electron rejection cut) [7].

\begin{tabular}{|c|c|c|c|}
\hline Category & $\epsilon_{\text {data }}(\%)$ & $\epsilon_{M C}(\%)$ & $\epsilon_{\text {data }} / \epsilon_{M C}$ \\
\hline \multicolumn{3}{|c|}{ All cuts except electron rejection (from $Z \rightarrow e e)$} \\
\hline 1 & $91.77 \pm 0.14$ & $92.43 \pm 0.07$ & $0.993 \pm 0.002$ \\
2 & $72.67 \pm 0.43$ & $71.89 \pm 0.08$ & $1.011 \pm 0.007$ \\
3 & $80.33 \pm 0.47$ & $80.04 \pm 0.18$ & $1.004 \pm 0.008$ \\
4 & $57.80 \pm 1.26$ & $55.09 \pm 0.15$ & $1.049 \pm 0.025$ \\
\hline \multicolumn{4}{|c|}{ Electron rejection cut (from $Z \rightarrow \mu \mu \gamma)$} \\
\hline 1 & $99.78_{-0.16}^{+0.13}$ & $99.59_{-0.17}^{+0.13}$ & $1.002_{-0.002}^{+0.002}$ \\
2 & $98.77_{-0.73}^{+0.59}$ & $97.70_{-0.37}^{+0.32}$ & $1.011_{-0.008}^{+0.007}$ \\
3 & $99.32_{-1.02}^{+0.51}$ & $99.29_{-0.42}^{+0.30}$ & $1.000_{-0.011}^{+0.006}$ \\
4 & $93.0_{-2.3}^{+2.1}$ & $93.34_{-0.86}^{+0.79}$ & $0.996_{-0.027}^{+0.024}$ \\
\hline
\end{tabular}

\subsection{Photon Energy Scale measurement}

The measured offset of the energy scale is defined as :

$$
s=\frac{E_{\text {measured }}^{\gamma}}{E_{\text {kinematics }}^{\gamma}}-1
$$

For $Z^{0} \rightarrow \mu \mu \gamma$ events, this can be written using the three-body decay kinematics, assuming the muon momenta are perfectly measured :

$$
s=\frac{m_{\mu \mu \gamma}^{2}-m_{\mu \mu}^{2}}{m_{Z^{0}}^{2}-m_{\mu \mu}^{2}}-1
$$

Distributions of $s$ in FSR events are presented in figure 4 . The simulation predicts $216 \pm 3$ events, where 193 events have been selected from in data. A binned Gaussian fit has been used to extract the scale in data, whereas an unbinned Crystal-Ball fit has been used for the simulation to optimize the use of the available statistics.

The extracted photon energy scale agrees with expectations at the $1 \%$ level in EB and $4 \%$ in EE. These numbers are within the estimated accuracy of the method, and are found to be consistent between three different methods. These results have been used in $Z \gamma$ and $W \gamma$ cross section measurements [3].

The energy scale in the endcaps is known with $1.5 \%$ uncertainty from the analysis of $Z^{0} \rightarrow e^{+} e^{-}$decays in 2010 [2].

\section{Conclusions \& Perspectives}

The $Z^{0} \rightarrow \mu \mu \gamma$ channel is the only available Standard Model source of pure high-energy photons. Three current uses of this channel within the CMS collaboration have been presented.

Up to now, photon studies relied mainly on $Z^{0}$ decays to electrons to examine in detail photon simulation, reconstruction and selection. With the available statistics recorded by CMS during $2011(\approx 4.5 / \mathrm{fb})$, the use of the $Z^{0} \rightarrow \mu \mu \gamma$ channel will be more effective than $Z^{0} \rightarrow e^{+} e^{-}$. This is of particular importance for $H \rightarrow \gamma \gamma$ searches. 


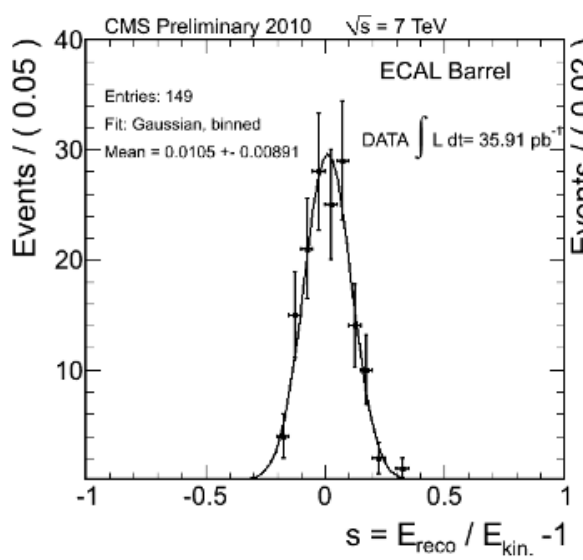

(a) DATA, ECAL barrel

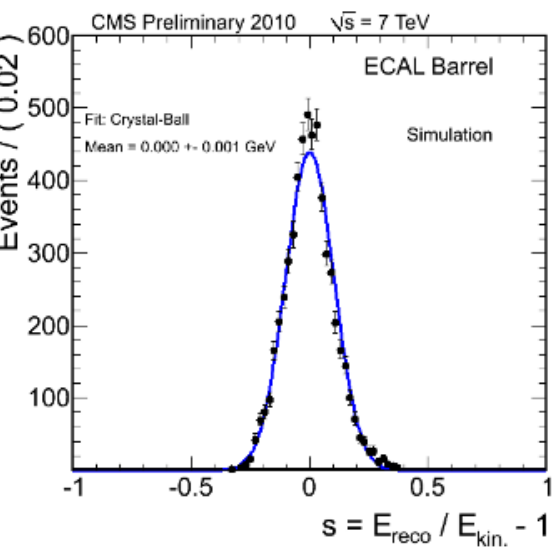

(b) MC, ECAL barrel

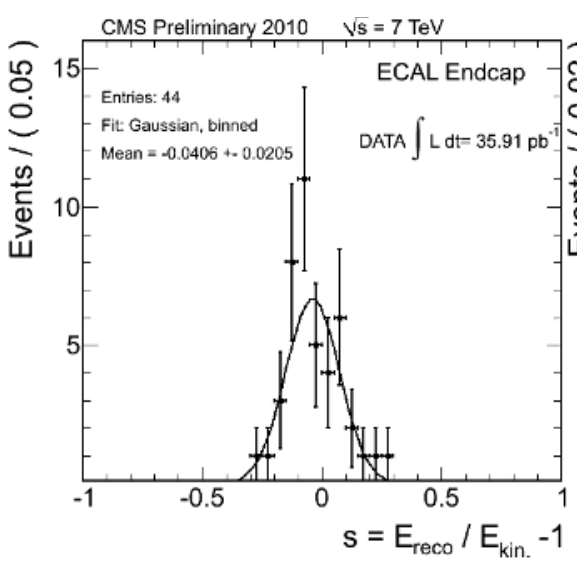

(c) DATA, ECAL endcaps

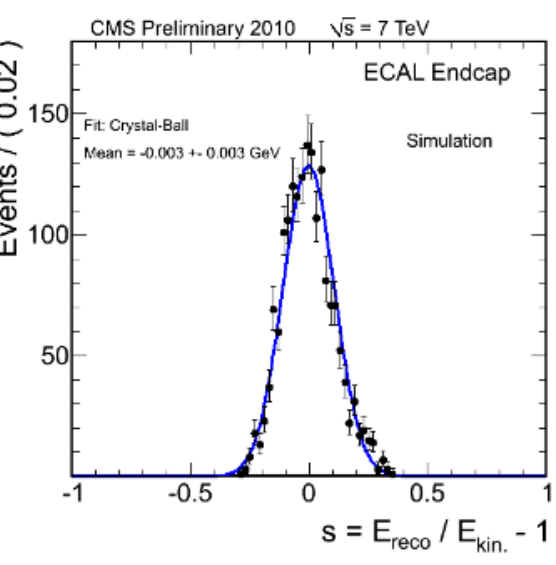

(d) MC, ECAL endcaps

Fig. 4: Measured offset of the photon energy scale 's', measured with FSR events in data collected in 2010 [2].

\section{References}

1. CMS Collaboration, J. Instrum. 2, (2008), S08004

2. CMS Collaboration, CMS ECAL 2010 performance results, (CMS-DP-2011-008)

3. CMS Collaboration, Phys. Lett. B701, (2011) 535-555

4. CMS Collaboration, CMS Physics Technical Design Report Volume I : Detector Performance and Software (CERN-LHCC-2006-001 ; CMS-TDR-008-1)

5. CMS Collaboration, Photon reconstruction and identification, (CMS-PAS-EGM-10-005)

6. CMS Collaboration, Search for a Higgs boson decaying into two photons in the CMS detector, (CMS-PAS-HIG11-010)

7. CMS Collaboration, Search for a Higgs boson decaying into two photons in the CMS detector, (CMS-PAS-HIG11-021) 